\title{
An Upper Limit on Gas Production from 3200 Phaethon
}

\author{
Paul A. Wiegert ${ }^{1}$ and Martin Houde \\ Department of Physics and Astronomy, The University of Western Ontario, London, \\ Canada N6A 3K7 \\ and Ruisheng Peng \\ Caltech Submillimeter Observatory, Hilo, HI 96720, USA
}

Received 01 Aug 2007; accepted 19 Dec 2007

Accepted for publication in Icarus 


\begin{abstract}
Asteroid 3200 Phaethon resembles a comet in some ways, including a highlyeccentric orbit ( $e \sim 0.89)$ and a strong associated meteor shower (the Geminids). Yet this object has never been observed to exhibit any cometary activity, i.e., gas production. We observed 3200 Phaethon with the Caltech Submillimeter Observatory on two occasions, once while it was near its closest approach to Earth as it neared perihelion, and another while it was further from Earth postperihelion. Observations of the $J=2 \rightarrow 1$ and $J=3 \rightarrow 2$ rotational transitions of ${ }^{12} \mathrm{CO}$, typically strong lines in comets and indicative of gas production, yielded no detection. Upper limits on the ${ }^{12} \mathrm{CO}$ production of $1.8 \times 10^{28}$ molecules $\mathrm{s}^{-1}$ and $7.6 \times 10^{28}$ molecules $\mathrm{s}^{-1}$ for Phaethon were determined on these two occasions.
\end{abstract}

Subject headings: asteroids — asteroid Phaethon — carbon monoxide - comets 


\section{Introduction}

Asteroid 3200 Phaethon was discovered by the Infrared Astronomical Satellite in 1983 (Green and Kowal 1983). Almost immediately thereafter its orbit was noted to be very similar to that of the Geminid meteor shower (Whipple 1983). This makes it the only asteroid linked to a strong meteor shower. A possible exception is asteroid 2003 EH1, which is associated with the Quadrantid shower (Jenniskens 2004; Williams et al. 2004; Wiegert and Brown 2005)) but which has not yet been observed near perihelion and thus whether it is asteroidal or cometary in nature remains unclear.

The link between Phathon and the Geminid meteor shower is also based on the closeness of their orbits, thus one could argue that they are simply aligned by coincidence. However, based on the very small difference between their orbits (see Table 1) and the absence of other objects on orbits nearby, Wiegert and Brown (2004) determined that probability of the orbital similarity of Phaethon and the Geminids being due to a chance alignment is less than one in a thousand. Though Phaethon's current activity (if any) is certainly low, intermittent or both, a study of the orbits of observed Geminids has indicated that they are consistent with cometary release no more than 2000 years ago and possibly

within the last 600 years (Gustafson 1989). Thus ongoing, perhaps sporadic, gas production by this object remains a possibility.

If 3200 Phaethon is not a comet, this does not mean it cannot be the parent body of the Geminids. A collision or impact might have released the material which formed the Geminid stream. A new rare population of "main-belt comets" has been uncovered in the

\footnotetext{
${ }^{1}$ Corresponding author: P. A. Wiegert, Department of Physics and Astronomy, The University of Western Ontario, London, Ontario, Canada N6A 3K7; e-mail: pwiegert@uwo.ca; Phone/fax: (519) 661-2111 x81327/(519) 661-2033.
} 
last several years (Hsieh and Jewitt 2006), bodies whose gas and dust production may be partly the result of collisions. However, the sublimation of volatiles from the body's surface also has a role to play, as evidenced by the seasonal cycle of activity that has been seen in one of them (133P/Elst-Pizarro) to date (Hsieh et al. 2007).

We note that Geminid meteors are much more durable than typical cometary meteors: studies of their ablation in Earth's atmosphere have shown that they have mean densities of $2.9 \mathrm{~g} \mathrm{~cm}^{-3}$ (Babadzhanov 2002), the highest of any of the streams measured. This may imply that the Geminids are made of relatively strong rocky asteroidal material rather than more porous and fragile cometary material. However, the question is complicated by the small (0.14 AU (Cook 1973)) perihelion distance of the Geminid stream. Smaller than is typical of comets, this low perihelion distance could result in extreme baking and sintering of the meteoroid particles, producing more durable meteoroids than might otherwise be expected of a cometary meteoroid stream.

Phaethon's association with a strong meteor shower and its highly eccentric orbit $(e \approx 0.89)$ make it a candidate cometary object. Yet in the over two decades since its discovery (which include many perihelion passages of its 1.43-year orbit), no cometary activity has ever been observed (Cochran and Barker 1984; Chamberlin et al. 1996; Hsieh and Jewitt 2005).

Phaethon's spectral type is B (Green et al. 1985), a primitive type associated with the outer portion of the main asteroid belt, though its albedo (0.11-0.17) is somewhat higher than would be expected of such a type (Veeder et al. 1984; Green et al. 1985), as was pointed out by Weissman et al. (2002). Visible and near IR spectroscopy of Phaethon support its identification as an asteroid rather than a comet nucleus (Licandro et al. 2007). Phaethon has been also linked with the smaller asteroid 2005 UD, with which it shares a bluish colour, an absence of dust production (Jewitt and Hsieh 2006) and an orbital 
similarity (Ohtsuka et al. 2006). Is Phaethon then an asteroid, and if so, how did it produce its meteor stream?

The question of the nature of this unusual object can only be answered by ongoing observations. In particular, observations which might reveal gas production by this body are the most important to undertake, as a positive detection would unequivocally identify this object as a comet, though its muted activity might imply it was extinct or dormant. Given the lack of previous detection of gas from Phaethon, its gas production rate is undoubtedly low, and observations should ideally be conducted near perihelion, or as close to it as can be managed given the interfering presence of the Sun.

In this paper we describe our attempts to measure gas production on Phaethon through the detection of $J=2 \rightarrow 1$ and $J=3 \rightarrow 2$ rotational transitions of the ${ }^{12} \mathrm{CO}$ molecule at the Caltech Submillimeter Observatory (CSO), located atop Mauna Kea, Hawaii. Observations in the $\mathrm{mm} / \mathrm{sub}-\mathrm{mm}$ range suffer the constraints of bad weather even more than traditional optical astronomy. This project arose from an idea to use time on the CSO that was unsuitable for projects requiring excellent atmospheric conditions for other purposes. The choice of Phaethon as a target, despite its lack of observed activity, was motivated by its meteor stream and the intermittency of activity seen in other comets eg. in the main-belt: ongoing monitoring of this object seems justifiable. The $J=2 \rightarrow 1$ and $J=3 \rightarrow 2$ rotational transitions of carbon monoxide $(\mathrm{CO})$ are strong in bright comets, though not perhaps ideal indicators of gas production for Phaethon. They were chosen as being among our best bets given our observational window during mediocre conditions $\left(\tau_{225}\right.$ GHz $\left.\sim 0.15\right)$. In fact our upper limit on gas production using these lines is comparable to that of a moderate-activity comet, and by observing as close to perihelion as possible, we hoped to catch Phaethon in outburst. Though our attempt was unsuccessful, only by ongoing observations will Phaethon's nature be clarified. 
We present the three spectra we obtained from corresponding sets of observations and discuss our results in the next sections.

\section{Observations}

The three sets of observations were taken at the Caltech Submillimeter Observatory, with the 200-300 GHz receiver on 5 November 2004 and 13 September 2005 for the ${ }^{12} \mathrm{CO}(J=2 \rightarrow 1)$ transition, and with the $300-400 \mathrm{GHz}$ receiver on 5 November 2004 for the ${ }^{12} \mathrm{CO}(J=3 \rightarrow 2)$ transition. All spectra were obtained with the high-resolution acousto-optics spectrometer (AOS) of $50 \mathrm{MHz}$ total bandwidth and approximately 100 $\mathrm{kHz}$ resolution. The CSO has a 10.4-meter dish and a beam diameter of approximately 30 and 20 arcseconds at the frequencies in question (i.e., approximately $230 \mathrm{GHz}$ and 345 $\mathrm{GHz}$ for ${ }^{12} \mathrm{CO} J=2 \rightarrow 1$ and $J=3 \rightarrow 2$, respectively). For all of our observations, the data were taken using the beam switching mode (i.e., by wobbling the secondary mirror) to allow for cancellation of sky background emission. While reducing the data we have set the receiver efficiencies to $70 \%$ for both transitions. This corresponds to situations where not only the main beam of the telescope probes the source, but also the sidelobes. This technique has been used by other investigators observing cometary lines at the CSO (eg. Bockelée-Morvan et al. (2000)). We assume that this would be applicable to our observations if there were a gas cloud surrounding 3200 Phaethon from which the sought transitions were detected (see Discussion below) .

For the observations obtained on 5 November 2004, 3200 Phaethon was 1.05 AU from Earth, allowing us a close look at the object as it passed, and 1.894 AU from the Sun, approaching perihelion. Its elevation angle varied from $54^{\circ}$ to $28^{\circ}$ in the morning sky, as it was moving rapidly towards the Sun. We obtained 50 minutes of observation (ON-source), but the $J=2 \rightarrow 1$ rotational transition line from ${ }^{12} \mathrm{CO}$ was not detected. Given mediocre 
sky conditions $\left(\tau_{225 \mathrm{GHz}} \simeq 0.15\right)$ and a system temperature of $T_{\text {sys }} \simeq 444 \mathrm{~K}$, we were able to integrate down to a noise floor of $14 \mathrm{mK}$ (with a velocity resolution of $\simeq 1 \mathrm{~km} \mathrm{~s}^{-1}$ after "smoothing" the spectrum to lower the noise level), and determine an upper limit $(3 \sigma)$ of approximately $T \simeq 42 \mathrm{mK}$ for the brightness temperature of the line. We also attempted to detect the $J=3 \rightarrow 2$ transition from ${ }^{12} \mathrm{CO}$ previously on the same night, when Phaethon was at an elevation of $70^{\circ}$ to $59^{\circ}$ with a similar outcome. For these observations, with a system temperature $T_{\text {sys }} \simeq 918 \mathrm{~K}$, the 48 minutes of ON-source integration yielded an upper limit of $T \simeq 69 \mathrm{mK}$ in a velocity resolution of $\simeq 0.7 \mathrm{~km} \mathrm{~s}^{-1}$. Our last set of data was obtained on 13 September 2005 when we again attempted to observe the $J=2 \rightarrow 1$ transition from ${ }^{12} \mathrm{CO}$ under comparable observing conditions (i.e., $\tau_{225 \mathrm{GHz}} \simeq 0.16$ and $\left.T_{\mathrm{sys}} \simeq 440 \mathrm{~K}\right)$. On that night, 3200 Phaethon was $1.904 \mathrm{AU}$ from Earth and 2.298 AU from the Sun approaching aphelion. Earlier observations nearer perihelion were impossible because Phaethon had been in the daytime sky for many months. This new set of data (50 minutes of integration ON-source), obtained at elevation angles ranging from $35^{\circ}$ to $70^{\circ}$, yielded no detection and an upper limit of approximately $T \simeq 48 \mathrm{mK}$, again with a velocity resolution of $\simeq 1 \mathrm{~km} \mathrm{~s}^{-1}$. The spectra that resulted from our observations are presented in Figure 1,

\section{Discussion}

The submillimeter rotational transitions of ${ }^{12} \mathrm{CO}$ have been studied in detail for comets and the strength of the lines can be linked to gas production rates (Crovisier and Le Bourlot 1983).

Assuming a lifetime of ${ }^{12} \mathrm{CO}$ against dissociation by solar UV radiation at $1 \mathrm{AU}$ of

$\tau=1.5 \times 10^{6} \mathrm{~s}($ Huebner and Carpenter (1979) $)$ from Crovisier and Le Bourlot (1983)) and assuming a gas expansion velocity $v_{\exp }=800 \mathrm{~m} / \mathrm{s}$ (the pre-perihelion value determined for 
Hyakutake from submillimeter observations by Biver et al. (1999)), any ${ }^{12} \mathrm{CO}$ cloud around 3200 Phaethon should have a diameter $\sim 2.4 \times 10^{6} \mathrm{~km}$, with an angular size of about 0.9 degree at 1 AU. A comparison of this angular size with the CSO main beam sizes quoted earlier for our observations justifies the efficiency adopted for our observations (i.e., 70\%).

Assuming fluorescence equilibrium and a Haser distribution (Haser 1957; Bertaux et al. 1998) with $\gamma_{\mathrm{CO}}=v_{\exp } \tau=1.2 \times 10^{6} \mathrm{~km}$, and using an emission constant $T_{B} \Delta v /\left\langle N_{\mathrm{CO}}\right\rangle$ of $3.65 \times 10^{-16} \mathrm{~K} \mathrm{~km} \mathrm{~s}^{-1}$ (molec. $\left.\mathrm{cm}^{-2}\right)^{-1}$ and $8.08 \times 10^{-16} \mathrm{~K} \mathrm{~km} \mathrm{~s}^{-1}\left(\text { molec. } \mathrm{cm}^{-2}\right)^{-1}$ for the $J=2 \rightarrow 1$ and $J=3 \rightarrow 2$ transitions, respectively (Crovisier and Le Bourlot) 1983), we can derive a corresponding upper limit on $Q_{\mathrm{CO}}$, the rate of ${ }^{12} \mathrm{CO}$ production, of approximately $1.8 \times 10^{28}$ molecules $\cdot \mathrm{s}^{-1}$ for 5 November 2004 (from the $J=3 \rightarrow 2$ transition). More precisely, at 1.05 AU (the distance between Earth and 3200 Phaethon during the corresponding observations) the dependence on the measured and assumed parameters can be expressed as

$$
Q_{\mathrm{CO}} \approx 3.60 \times 10^{28}\left(\frac{v_{\exp }}{800 \mathrm{~m} / \mathrm{s}}\right)^{2}\left(\frac{T_{B}}{42 \mathrm{mK}}\right) \text { molecules } \cdot \mathrm{s}^{-1}
$$

for the $J=2 \rightarrow 1$ transition, and

$$
Q_{\mathrm{CO}} \approx 1.77 \times 10^{28}\left(\frac{v_{\exp }}{800 \mathrm{~m} / \mathrm{s}}\right)^{2}\left(\frac{T_{B}}{69 \mathrm{mK}}\right) \text { molecules } \cdot \mathrm{s}^{-1}
$$

for the $J=3 \rightarrow 2$ transition. Using an equation similar to equation (1) for a distance of $1.904 \mathrm{AU}$ we also establish an upper limit of $Q_{\mathrm{CO}} \approx 7.6 \times 10^{28}$ molecules $\cdot \mathrm{s}^{-1}$ for 13 September 2005.

These upper limits are lower than production rates observed for very bright comets at the same heliocentric distance, e.g., C $1995 \mathrm{O} 1$ (Hale-Bopp), $Q_{\mathrm{CO}}=2.07 \times 10^{30}$ molecules $\cdot \mathrm{s}^{-1}$ (DiSanti et al. 2001). The limits are comparable to the production rate of $\mathrm{C} / 1996 \mathrm{~B} 2$ 
Hyakutake, $Q_{\mathrm{CO}} \approx 5 \times 10^{28}$ molecules $\cdot \mathrm{s}^{-1}$ near $1 \mathrm{AU}$ (Biver et al. 1999), though it is a long-period comet and expected to be rich in ices.

Comet 2P/Encke, an old low-activity comet, might provide a better benchmark. Though its carbon monoxide production has not been measured to our knowledge, we can estimate this from its $\mathrm{H}_{2} \mathrm{O}$ production rate, which has measured at 0.8 to $2.6 \times 10^{28}$ molecules $\mathrm{s}^{-1}$ at similar heliocentric distances (Mäkinen et al. 2001). The ${ }^{12} \mathrm{CO}$ to $\mathrm{H}_{2} \mathrm{O}$ ratio in comets is variable, ranging from $0.5 \%$ to $22 \%$ (Biver et al. 1999; DiSanti et al. 2001; Mumma et al. 2001a,b.,c); DiSanti et al. 2002; Feldman et al. 2002; DiSanti et al. 2003, 2007). Taking the ratio to be $5 \%$, we can derive a corresponding expected ${ }^{12} \mathrm{CO}$ production rate of $0.4 \times 10^{27}$ to $1.3 \times 10^{27}$ molecules $\mathrm{s}^{-1}$ for comet Encke. Though a nearly-extinct comet could produce ${ }^{12} \mathrm{CO}$ at even lower rates, this presents us with a target measurement sensitivity of $Q_{\mathrm{CO}} \sim 10^{27}$ molecules s ${ }^{-1}$.

In view of these numbers it will be necessary to make deeper observations of 3200 Phaethon in order to detect any ${ }^{12} \mathrm{CO}$ production or to reduce significantly our derived upper limits. It follows that we need to increase our sensitivity by at least an order of magnitude. The results discussed here were obtained under relatively poor sky conditions and improving these limits should be feasible with a combination of longer exposure times and better observing conditions (e.g., $\tau_{225 \mathrm{GHz}} \simeq 0.05$ ), as is relatively common (20\% to $25 \%$ of the time) on Mauna Kea. The sensitivity can also be increased by observing Phaethon when it is closest to Earth, as the detection limit drops approximately linearly with the Earth-comet distance. Observations of this object during its upcoming close approach to Earth $(\lesssim 0.13$ AU in December 2007) would thus be particularly recommended.

M.H.'s research is funded through the NSERC Discovery Grant, Canada Research Chair, Canada Foundation for Innovation, Ontario Innovation Trust, and the University of Western Ontario's Academic Development Fund programs. This work was supported in 
part by the Natural Sciences and Engineering Research Council of Canada. The Caltech Submillimeter Observatory is funded by the NSF through contract AST 05-40882. 


\section{REFERENCES}

Babadzhanov, P. B. 2002. Fragmentation and densities of meteoroids. A\&A 384, 317-321.

Bertaux, J. L., J. Costa, E. Quémerais, R. Lallement, M. Berthé, E. Kyrölä, W. Schmidt, T. Summanen, T. Makinen, and C. Goukenleuque. 1998. Lyman-alpha observations of comet Hyakutake with SWAN on SOHO. Planet. Space Sci. 46, 555-568.

Biver, N., D. Bockelée-Morvan, J. Crovisier, J. K. Davies, H. E. Matthews, J. E. Wink, H. Rauer, P. Colom, W. R. F. Dent, D. Despois, R. Moreno, G. Paubert, D. Jewitt, and M. Senay. 1999. Spectroscopic Monitoring of Comet C/1996 B2 (Hyakutake) with the JCMT and IRAM Radio Telescopes. AJ 118, 1850-1872.

Bockelée-Morvan, D., D. C. Lis, J. E. Wink, D. Despois, J. Crovisier, R. Bachiller, D. J. Benford, N. Biver, P. Colom, J. K. Davies, E. Gérard, B. Germain, M. Houde, D. Mehringer, R. Moreno, G. Paubert, T. G. Phillips, and H. Rauer. 2000. New molecules found in comet C/1995 O1 (Hale-Bopp). Investigating the link between cometary and interstellar material. A\&A 353, 1101-1114.

Chamberlin, A. B., L. McFadden, R. Schulz, D. G. Schleicher, and S. J. Bus. 1996. 4015 Wilson-Harrington, 2201 Oljato, and 3200 Phaethon: Search for CN Emission. Icarus 119, 173-181.

Cochran, A. L. and E. S. Barker. 1984. Minor planet 1983TB - A dead comet? Icarus 59, 296-300.

Cook, A. F. 1973. A working list of meteor streams. In C. L. Hemenway, P. M. Millman, and A. F. Cook, (Eds.), Evolutionary and Physical Properties of Meteoroids. NASA, Washington, pp. 183-191. 
Crovisier, J. and J. Le Bourlot. 1983. Infrared and microwave fluorescence of carbon monoxide in comets. $A \mathscr{\mho} A \mathbf{1 2 3}, 61-66$.

DiSanti, M. A., M. J. Mumma, N. Dello Russo, and K. Magee-Sauer. 2001. Carbon Monoxide Production and Excitation in Comet C/1995 O1 (Hale-Bopp): Isolation of Native and Distributed CO Sources. Icarus 153, 361-390.

DiSanti, M. A., N. Dello Russo, K. Magee-Sauer, E. L. Gibb, D. C. Reuter, and M. J. Mumma. 2002. $\mathrm{CO}, \mathrm{H}_{2} \mathrm{CO}$, and $\mathrm{CH}_{3} \mathrm{OH}$ in comet $2002 \mathrm{C} 1$ Ikeya-Zhang. In B. Warmbein, (Ed.), Asteroids, Comets, and Meteors: ACM 2002, Vol. 500 of ESA Special Publication, pp. 571-574.

DiSanti, M. A., M. J. Mumma, N. Dello Russo, K. Magee-Sauer, and D. M. Griep. 2003. Evidence for a dominant native source of carbon monoxide in Comet C/1996 B2 (Hyakutake). Journal of Geophysical Research (Planets) 108, 15-1.

DiSanti, M. A., W. M. Anderson, G. L. Villanueva, B. P. Bonev, K. Magee-Sauer, E. L. Gibb, and M. J. Mumma. 2007. Depleted Carbon Monoxide in Fragment C of the Jupiter-Family Comet 73P/Schwassmann-Wachmann 3. ApJ 661, L101-L104.

Feldman, P. D., H. A. Weaver, and E. B. Burgh. 2002. Far Ultraviolet Spectroscopic Explorer Observations of $\mathrm{CO}$ and $\mathrm{H}_{2}$ Emission in Comet $\mathrm{C} / 2001$ A2 (LINEAR). ApJ 576, L91-L94.

Green, S. and C. Kowal. 1983. 1983 TB. IAU Circular 3878, 1.

Green, S. F., A. J. Meadows, and J. K. Davies. June 1985. Infrared observations of the extinct cometary candidate minor planet (3200) 1983TB. MNRAS 214, 29P-36P.

Gustafson, B. A. S. 1989. Geminid meteoroids traced to cometary activity on Phaethon. AधA 225, 533-540. 
Haser, L. 1957. Distribution d'intensité dans la tête d'une comète. Bulletin de la Société Royale des Sciences de Liège 43, 740-750.

Hsieh, H. H. and D. Jewitt. 2005. Search for Activity in 3200 Phaethon. ApJ 624, 1093-1096.

Hsieh, H. H. and D. Jewitt. 2006. A Population of Comets in the Main Asteroid Belt. Science 312, 561-563.

Hsieh, H. H., D. Jewitt, S. C. Lowry, and C. Snodgrass. 2007. The Return of Activity in Comet 133P/Elst-Pizarro. In AAS/Division for Planetary Sciences Meeting Abstracts, Vol. 39 of AAS/Division for Planetary Sciences Meeting Abstracts, p. \#59.01.

Huebner, W. F. and C. W. Carpenter. 1979. Solar photo rate coefficients. NASA STI/Recon Technical Report N 80.

Jenniskens, P. 2004. 2003 EH1 is the Quadrantid shower parent comet. AJ 127, 3018-3022.

Jewitt, D. and H. Hsieh. 2006. Physical Observations of 2005 UD: A Mini-Phaethon. AJ 132, 1624-1629.

Licandro, J., H. Campins, T. Mothé-Diniz, N. Pinilla-Alonso, and J. de León. 2007. The nature of comet-asteroid transition object (3200) Phaethon. A\&A 461, 751-757.

Mäkinen, J. T. T., J. Silén, W. Schmidt, E. Kyrölä, T. Summanen, J.-L. Bertaux, E. Quémerais, and R. Lallement. 2001. Water Production of Comets 2P/Encke and 81P/Wild 2 Derived from SWAN Observations during the 1997 Apparition. Icarus 152, 268-274.

Mumma, M. J., N. Dello Russo, M. A. DiSanti, K. Magee-Sauer, R. Novak, A. Conrad, and F. Chaffee. 2001a. Comet C/1999 T1 (McNaught-Hartley). IAU Circ. 7578. 
Mumma, M. J., N. Dello Russo, M. A. DiSanti, K. Magee-Sauer, R. E. Novak, S. Brittain, T. Rettig, I. S. McLean, D. C. Reuter, and L.-H. Xu. 2001b. Organic Composition of C/1999 S4 (LINEAR): A Comet Formed Near Jupiter? Science 292, 1334-1339.

Mumma, M. J., I. S. McLean, M. A. DiSanti, J. E. Larkin, N. D. Russo, K. Magee-Sauer, E. E. Becklin, T. Bida, F. Chaffee, A. R. Conrad, D. F. Figer, A. M. Gilbert, J. R. Graham, N. A. Levenson, R. E. Novak, D. C. Reuter, H. I. Teplitz, M. K. Wilcox, and L.-H. Xu. 2001c. A Survey of Organic Volatile Species in Comet C/1999 H1 (Lee) Using NIRSPEC at the Keck Observatory. ApJ 546, 1183-1193.

Ohtsuka, K., T. Sekiguchi, D. Kinoshita, J.-I. Watanabe, T. Ito, H. Arakida, and T. Kasuga. 2006. Apollo asteroid 2005 UD: split nucleus of (3200) Phaethon? A\&A 450, L25-L28.

Veeder, G. J., C. Kowal, and D. L. Matson. March 1984. The Earth-Crossing Asteroid 1983 TB. In Lunar and Planetary Institute Conference Abstracts, Vol. 15 of Lunar and Planetary Institute Conference Abstracts, pp. 878-879.

Weissman, P. R., W. F. Bottke, Jr., and H. F. Levison. 2002. Evolution of Comets into Asteroids. Asteroids III pp. 669-686.

Whipple, F. L. 1983. 1983 TB and the Geminid Meteors. IAU Circular 3881, 1.

Wiegert, P. and P. Brown. 2004. The problem of linking minor meteor showers to their parent bodies: initial considerations. Earth, Moon, and Planets 95, 19-26.

Wiegert, P. and P. Brown. 2005. The Quadrantid meteoroid complex. Icarus 179, 139-157.

Williams, I. P., G. O. Ryabova, A. P. Baturin, and A. M. Chernitsov. 2004. The parent of the Quadrantid meteoroid stream and asteroid 2003 EH1. MNRAS 355, 1171-1181. 
This manuscript was prepared with the AAS IATEX macros v5.2. 


\begin{tabular}{lccccccc}
\hline & $a(\mathrm{AU})$ & $e$ & $q(\mathrm{AU})$ & $i(\mathrm{deg})$ & $\Omega(\mathrm{deg})$ & $\omega(\mathrm{deg})$ & $T_{J}$ \\
\hline 3200 Phaethon & 1.27 & 0.890 & 0.140 & 22.2 & 265.4 & 322.0 & 4.51 \\
Geminids & 1.36 & 0.896 & 0.142 & 23.6 & 261.0 & 324.3 & 4.23 \\
\hline
\end{tabular}

Table 1: Standard orbital elements of 3200 Phaethon (NeoDys website http://newton.dm.unipi.it/cgi-bin/neodys/neoibo) and the Geminid meteor shower (Cook 1973). 


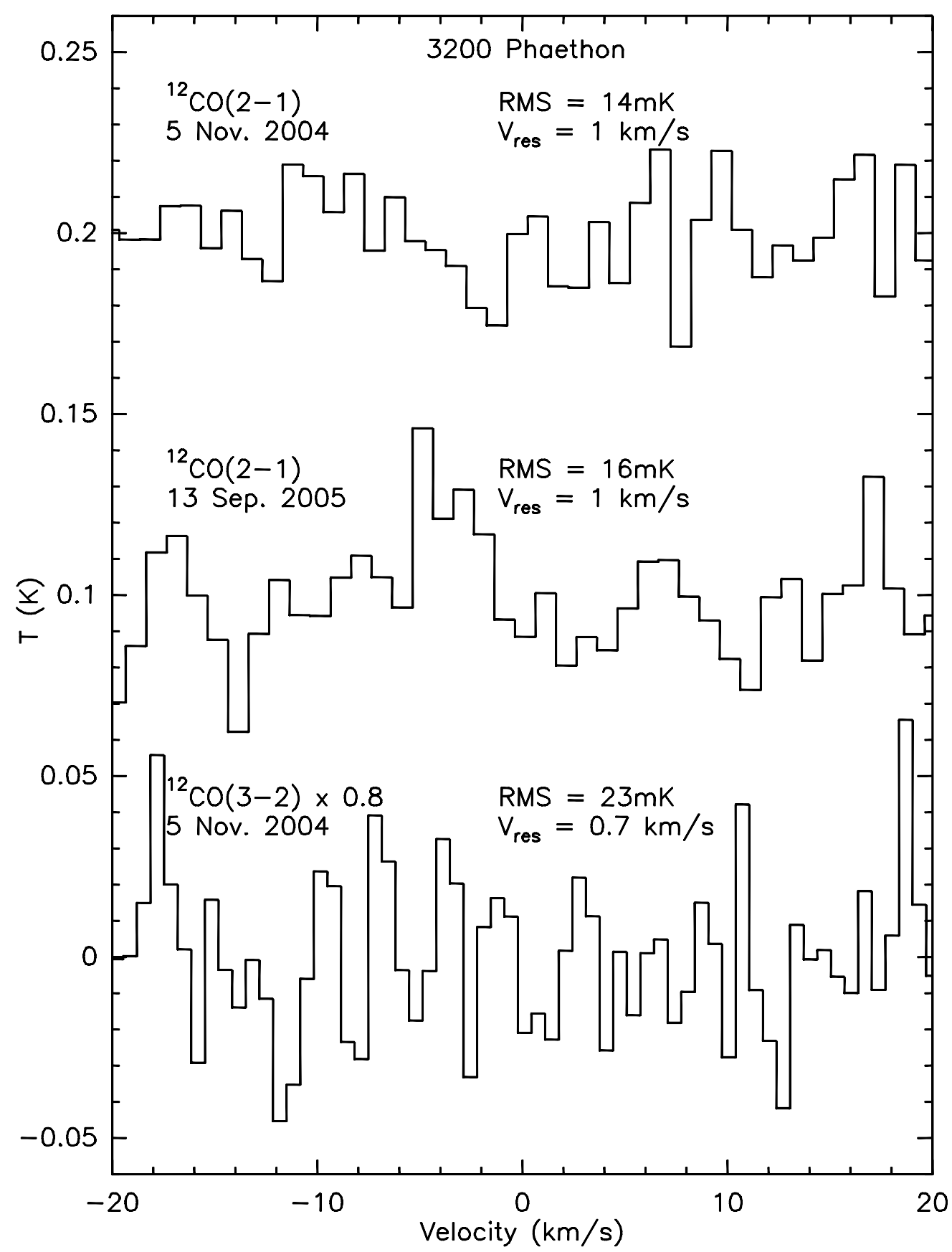

Fig. 1.- The three spectra that resulted from our observations. The top two are for our attempts at a detection of the $J=2 \rightarrow 1$ transition of ${ }^{12} \mathrm{CO}$, while the the bottom spectrum corresponds to our $J=3 \rightarrow 2$ observations. 